\title{
Les effets des mouvements oculaires bilatéraux sur la cohérence EEG lors du rappel d'un souvenir plaisant
}

\author{
Brandon Keller \\ Larry Stevens \\ Colleen Lui \\ James Murray \\ Matthew Yaggie \\ Université de l'Arizona du nord
}

\begin{abstract}
Dans une enquête sur le modèle de cohérence interhémisphérique $(\mathrm{Clh})$ dans la désensibilisation et le retraitement par les mouvements oculaires (EMDR) et les effets des mouvements oculaires bilatéraux (MOB), 30 sujets ont été exposés à un point fixe, à un point clignotant vert/rouge ou à des MOB alternés pendant la visualisation d'un souvenir agréable. On a mesuré ensuite les électroencéphalogrammes (EEG) lors d'une étape de traitement où les participants avaient les yeux fermés. Les analyses n'ont pas révélé d'amélioration significative de la $\mathrm{Cl}$ h pour la condition $\mathrm{MOB}$, mais des augmentations importantes dans la cohérence MOB intrahémisphérique des EEG des ondes delta et bêta basses dans les zones frontales droites et gauches, respectivement, et une augmentation tendancielle dans la cohérence MOB des ondes bêta basses de la zone frontale droite. On a eu recours à la neuro-imagerie LORETA afin de visualiser les changements significatifs d'amplitude correspondants aux effets de cohérence observés. Nous exposons ici la signification fonctionnelle de ces effets de cohérence intrahémisphérique et nous suggérons d'étendre le modèle $\mathrm{Clh}$ à la cohérence corticale.
\end{abstract}

Mots-clés : EMDR ; mouvements oculaires bilatéraux ; EEG ; cohérence ; mémoire épisodique ; ESPT

$\mathbf{L}$ a désensibilisation et le retraitement par les mouvements oculaires (EMDR) a été reconnue pour être une approche thérapeutique efficace dans le traitement des effets des souvenirs traumatiques (Association psychiatrique américaine, 2004 ; Bisson et coll., 2007 ; Cukor, Olden, Lee \& Difede, 2010 ; Lamprecht et coll., 2004). Malgré ces soutiens positifs, les comparaisons empiriques de l'EMDR avec d'autres traitements populaires comme l'exposition prolongée, l'entraînement à l'inoculation du stress, la thérapie cognitive comportementale et la thérapie par relaxation, se sont surtout avérées équivoques : certaines études ont en effet montré que l'EMDR était plus efficace, d'autres qu'elle l'était moins et d'autres encore qu'elle était équivalente (p. ex. Davidson \& Parker, 2001 ; Devilly \& Spence, 1999 ;
Ironson, Freund, Strauss \& Williams, 2002 ; Power et coll., 2002 ; Lee, Gavriel, Drummond, Richards \& Greenwald, 2002 ; Rothbaum, Astin \& Marsteller, 2005 ; Taylor et coll., 2003). Néanmoins, l'EMDR reste l'un des traitements les plus populaires de l'état de stress post-traumatique (ESPT ; Pagani, Högberg, Fernandez \& Siracusano, 2013). Une meilleure compréhension des composantes de cette technique thérapeutique pourrait peut-être conduire à de subtiles améliorations du protocole, qui produiraient des résultats encore supérieurs et une meilleure assistance aux victimes de trauma.

Une des composantes centrales de l'EMDR, qui la distingue d'autres stratégies de traitement du trauma, est l'utilisation de la stimulation bilatérale lors de la visualisation d'événements traumatiques cibles (Shapiro,

This article originally appeared as Keller, B., Stevens, L., Lui, C., Murray, J., \& Yaggie, M. (2014). The Effects of Bilateral Eye Movements on EEG Coherence When Recalling a Pleasant Memory. Journal of EMDR Practice and Research, 8(3), $113-128$.

Translated by François Mousnier-Lompré. 
1989 ; Shapiro \& Maxfield, 2002 ; Solomon \& Shapiro, 2008). Le modèle de traitement adaptatif de l'information (TAI) de Shapiro (Shapiro, 2001 ; Solomon $\&$ Shapiro, 2008) postule que la stimulation bilatérale active plus de réseaux neuronaux isolés pour permettre la connexion entre des informations dissociées et des événements traumatiques, facilitant ainsi le retraitement de ces événements et leur désensibilisation finale. Shapiro (1989) utilisait initialement la stimulation bilatérale visuelle au moyen d'un mouvement latéral des doigts à travers le champ visuel du patient, à une cadence approximative de deux mouvements oculaires par seconde. Toutefois, depuis la découverte des contributions de cette composante au traitement des souvenirs traumatiques, les stimulations bilatérales auditive et kinesthésique ont également été employées, avec des résultats équivalents (Harper, 2012).

Bien que de nombreuses théories aient été proposées pour expliquer les apports de la stimulation bilatérale au traitement et à la dépotentialisation de souvenirs traumatiques (Bergmann, 2008), les mécanismes d'action de cette composante n'ont pas, à ce jour, été définitivement expliqués. Un des modèles d'orientation plutôt neurobiologique pour expliquer les effets de la stimulation bilatérale sur l'ESPT, le modèle du couplage amygdale-cortex cingulaire antérieur (CCA)/cortex préfrontal (CPF), est confronté à un nombre croissant de preuves (a) d'une suractivation de processus amygdaliens impliqués dans l'expérience émotionnelle des événements traumatiques, combinée avec (b) une désactivation ou un découplage des fonctions du CCA et du CPF médian qui, autrement, permettraient le traitement cognitif et la dépotentialisation de ces événements dans l'ESPT (Francati, Vermetten \& Bremner, 2007). Faisant une analyse encore plus réductionniste, ce modèle de la symptomatologie ESPT émet en outre l'hypothèse que les souvenirs traumatiques sont verrouillés dans des réseaux synaptiques en résonance mutuelle de récepteurs alpha-amino-3-hydroxy-5-méthyl-4-isoxazole (récepteurs AMPA) surpotentialisés dans l'amygdale (Harper, Rasolkhani-Kahophorn \& Drozd, 2009). (c) Ce traitement pathologique du trauma est pour l'essentiel réordonné au cours de la ré-expérience de l'événement par les stimulations sensorielles bilatérales qui apportent la stimulation tétanique à basse fréquence nécessaire pour dépotentialiser ces récepteurs AMPA et, subséquemment, les souvenirs amygdaliens verrouillés. (d) Cette dépotentialisation des réseaux neuraux verrouillés permet ensuite à ces souvenirs émotionnels de se diffuser dans les régions $C A$ et $\mathrm{CPF}$ où ils peuvent être retraités plus naturellement et de façon plus cognitive. Des éléments de ce modèle ont reçu un certain soutien d'études portant sur la neuroimagerie animale et humaine (pour une revue complète de ces publications, voir Pagani et coll., 2013).

F. Shapiro (1989) avait très tôt fait l'hypothèse que la stimulation visuelle bilatérale alternée employée dans l'EMDR activait des réseaux de neurones des deux côtés du cerveau et permettait aux réseaux jusqu'ici dissociés de se lier aux événements traumatiques ciblés en vue de leur retraitement final. Proposée initialement par Servan-Schreiber (2000) et élaborée empiriquement par Christman et coll. (Christman, Garvey, Propper \& Phaneuf, 2003; Christman, Propper \& Brown, 2006; Christman, Propper \& Dion, 2004; Propper \& Christman, 2008), cette hypothèse de la connectivité interhémisphérique visant à expliquer les effets de la stimulation bilatérale sur la récupération de la mémoire épisodique a fait l'objet d'un grand nombre d'investigations. Si cette hypothèse est correcte, deux résultats doivent apparaître : (a) la récupération mnésique doit s'améliorer pendant, ou immédiatement après, la stimulation bilatérale et (b) les mesures des connexions interhémisphériques doivent montrer une augmentation de celles-ci à la suite de la stimulation bilatérale. À ce jour, les études ont apporté un certain appui empirique à ces deux prévisions.

Par exemple, Christman et coll. (2003) ont constaté une amélioration de la reconnaissance des mots et de la récupération des souvenirs autobiographiques à la suite d'une séquence de 30 secondes de mouvements oculaires horizontaux alternés. Ces résultats ont été appuyés par des études antérieures sur la préférence latérale (en tant que représentation d'interaction interhémisphérique) et par les effets d'une présentation séquentielle de stimuli visuels bilatéraux sur la mémoire (Christman \& Propper, 2001). D’autres recherches, montrant une amélioration des mesures comportementales d'interaction interhémisphérique et de la créativité à la suite de mouvements oculaires bilatéraux (MOB ; Shobe, Ross \& Fleck, 2009), une amélioration du rappel et de la précision du souvenir lors du récit d'un événement visuel après les MOB (Parker, Buckley \& Dagnall, 2009), une amélioration de la récupération des souvenirs (Christman et coll., 2003 ; Lyle, Logan \& Roediger, 2008), une détérioration de la mémoire épisodique à la suite d'une commissurotomie (Cronin-Golomb, Gabrieli $\&$ Keane, 1996), et d'autres études (voir Propper \& Christman, 2008, pour une revue complète de cette littérature) appuient fortement l'hypothèse d'une meilleure récupération de la mémoire de type épisodique à la suite de la présentation de mouvements oculaires bilatéraux alternés. 
La littérature est cependant plus rare et plus ambiguë concernant les effets de la stimulation bilatérale sur des mesures directes de la connectivité interhémisphérique. Un des moyens de mesure de la connectivité fonctionnelle estl'électroencéphalographie(EEG)dela cohérence interhémisphérique (CIh). La cohérence EEG est une mesure quantitative des formes d'onde EEG ou de la cohérence de phase entre deux zones différentes du cuir chevelu (Nunez et coll., 1997). Mathématiquement, les valeurs de cohérence représentent la fonction de densité spectrale croisée de la forme d'onde EEG normalisée par les spectres de puissance et sont représentées par une fonction de corrélation au carré possédant une magnitude comprise entre 0 et \pm 1 . Ainsi, la cohérence peut être interprétée comme la communication ou la connectivité fonctionnelles entre deux sites d'enregistrement, et une cohérence plus élevée correspond à une coopération et à une synchronisation plus importantes entre des régions cérébrales mesurées à une fréquence spécifiée (Knott, LaBelle, Jones \& Mahoney, 2002 ; Nunez et coll., 1997 ; Weiss \& Mueller, 2003). Bergmann (2008) affirme que les oscillations neurales synchronisées, indexées largement par la cohérence EEG corticale, sont à la base de la perception et du fonctionnement humains. Si les sites d'enregistrement sélectionnés sont des sites homologues sur les côtés opposés du cortex (p. ex. électrodes F7 et F8 ; voir Figure 1), la cohérence EEG est alors une mesure idéale de la connectivité neuro-électrique interhémisphérique.

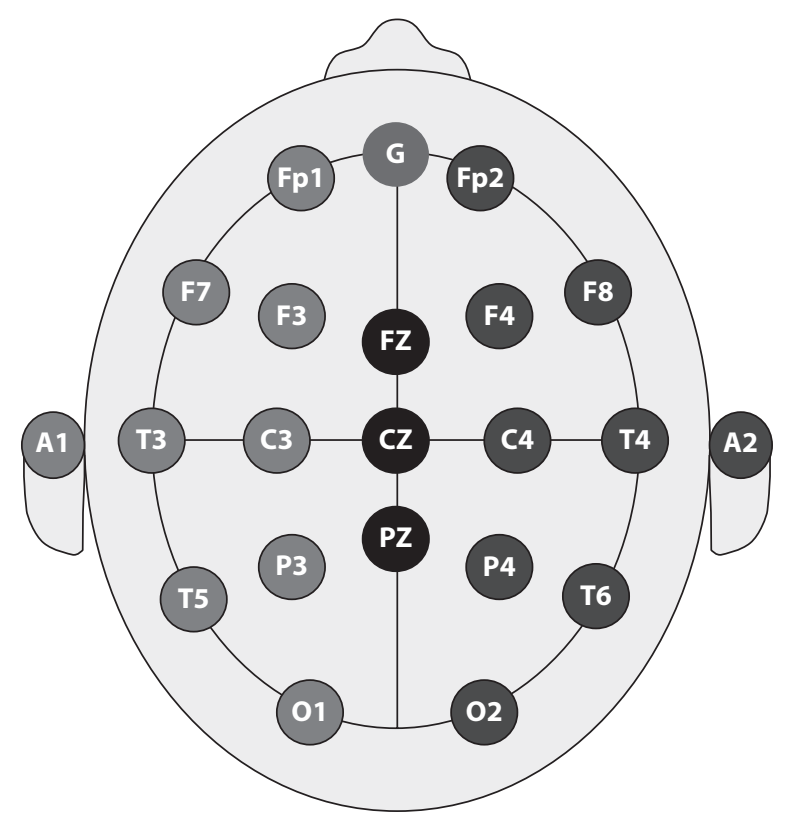

FIGURE 1. Schéma du système international 10-20 pour le placement des électrodes EEG.
Cependant, deux études seulement ont été publiées à ce jour sur les effets des mouvements oculaires horizontaux alternés sur la CIh. Propper, Pierce, Geisler, Christman et Bellorado (2007) ont examiné la CIh à partir de deux sites d'électrode frontaux bilatéraux (Fp1 et $\mathrm{Fp} 2)$ avant et après une présentation de 30 secondes, soit d'un point en mouvement provoquant deux mouvements oculaires alternés par seconde, soit d'un point stationnaire rouge/vert clignotant deux fois par seconde, dans un modèle intergroupes. Leurs résultats, en contradiction avec leurs prédictions initiales, ont montré une diminution de la cohérence dans la bande de fréquence EEG gamma $(35-54 \mathrm{~Hz})$ et aucun effet sur les bandes thêta (4-8 Hz) et alpha (8-13), dans la condition du stimulus en mouvement par rapport à la condition de contrôle du point clignotant. En dépit de l'interprétation post hoc, par ces auteurs, de ces résultats surprenants, selon laquelle des changements importants dans la coordination interhémisphérique sont induits par les mouvements oculaires, cette étude contient plusieurs points critiques rendant discutable sa pertinence pour la pratique EMDR et pour la connectivité interhémisphérique liée à une tâche. Premièrement, les auteurs ont sélectionné deux sites d'enregistrement au-dessus des muscles frontaux, sites très sensibles aux artéfacts musculaires résiduels induits par les mouvements oculaires, et ils ont enregistré à partir de ces sites au cours des 3 secondes de déclenchement du stimulus, contaminant potentiellement les enregistrements EEG avec des artéfacts musculaires qui peuvent apparaitre dans les bandes gamma et delta (ils ne disent rien de la bande delta et ils ont dû par ailleurs exclure de l'analyse quelque $20 \%$ de leur groupe de participants initial, en raison d'enregistrements EEG confus et inutilisables.) Deuxièmement, leurs valeurs de cohérence EEG, particulièrement pour alpha et thêta, étaient très élevées, avoisinant 1,00 même avant les stimulations, suggérant un effet plafond qui réduisait la probabilité d'obtenir des changements de cohérence importants et significatifs. Et, troisièmement, on ne demandait aux sujets aucune tâche de rappel de mémoire épisodique au cours des conditions de mouvements oculaires, comme cela se produit en EMDR, privant l'étude d'une activité de tâche spécifique servant de base d'examen de la coordination des réseaux neuronaux.

La seconde étude de Samara, Elzinga, Slagter et Nieuwenhuis (2011), bien conçue et étroitement contrôlée, calculait la cohérence EEG, de phase et d'amplitude, sur la totalité du cuir chevelu avant la participation à une tâche neutre ou émotionnelle de rappel de mots, dans les mêmes conditions de 
contrôle et de MOB que Propper et coll. (2007) mais en utilisant une conception intrasujet plus puissante que la leur. En outre, ces chercheurs ont enregistré des électrooculogrammes afin de vérifier les MOB et ils ont minutieusement créé des artéfacts visuels et statistiques dans les données EEG afin d'éliminer ensuite le bruit et les artéfacts musculaires de leurs enregistrements. Malheureusement pour le modèle CIh et à la suite d'analyses de rigueur multiples et réduites, Samara et coll. n'ont constaté à l'EEG aucun changement, régulier ou prévu, au niveau de la phase ou de l'amplitude de la cohérence, entre les conditions pré- et post-MOB ou au cours des mouvements oculaires. Ils ont observé une diminution importante de la cohérence d'amplitude alpha, bilatéralement, pour les électrodes F7-F8 dans la condition MOB, mais une augmentation de la cohérence d'amplitude alpha pour ces électrodes dans la condition de contrôle. Bien qu'ils n'aient découvert une amélioration significative dans le rappel des mots émotionnels que pour la condition MOB, il n'y avait pas de corrélation significative entre la cohérence et le rappel de mots. À première vue, cette étude remet considérablement en question l'efficacité déclarée de l'EMDR et plus spécifiquement en ce qui concerne les améliorations bien documentées de la récupération de souvenirs à la suite des MOB.

Cependant, l'étude présentait une lacune importante, que les auteurs ont reconnue : l'absence d'une véritable tâche de récupération de souvenirs épisodiques (Tulving, 1985) dans la composante des mouvements oculaires de l'étude. Effectivement, non seulement la tâche cognitive utilisée dans cette étude était une tâche de rappel de la mémoire sémantique, mais un film "documentaire neutre " de 30 minutes était projeté à la suite de la présentation des mots et avant l'enregistrement EEG et la mise en route des MOB. Comme indiqué précédemment, le maintien de l'événement traumatique dans la mémoire de travail pendant les MOB est une caractéristique importante et unique de la thérapie EMDR. En réalité, très peu des études publiées sur les effets des MOB sur la récupération de souvenirs ont fait appel à des tâches portant sur des souvenirs épisodiques significatifs sur le plan personnel et ont demandé aux participants de rester concentrés sur ces souvenirs dans la mémoire de travail au cours de la stimulation bilatérale. Deux études font pourtant exception : la seconde expérience conduite par Christman et coll. (2003) qui a constaté une amélioration sélective de souvenirs épisodiques réels à la suite des MOB et l'étude sur la visualisation de souvenirs d'enfance pendant les MOB, également conduite par Christman et coll. (2006), qui a constaté que l'amnésie de l'enfance prenait fin plus tôt. Aucune de ces études, cependant, ne portait sur la mesure de la cohérence EEG.

Le modèle CIh concernant les effets de l'EMDR sur le retraitement des souvenirs traumatiques ne sera testable que lorsqu'on mesurera la CIh pendant ou immédiatement après les stimulations bilatérales pendant lesquelles le sujet se concentrera sur des souvenirs épisodiques significatifs pour lui. De plus, dans la mesure où l'activation de réseaux neuronaux éloignés, telle que la propose le modèle TAI, peut également avoir lieu à l'intérieur des hémisphères, notre enquête a examiné la cohérence intrahémisphérique. L'étude ouvre ce domaine d'investigation par l'enregistrement et l'analyse de la cohérence EEG multicanal inter- et intrahémisphérique après les MOB, sur deux conditions de contrôle, pendant la concentration sur des souvenirs épisodiques positifs ayant une signification personnelle pour le sujet. Des souvenirs positifs ont été utilisés dans cette enquête, afin d'éviter le risque potentiel de retraumatiser notre jeune échantillon non clinique, de faciliter l'approbation du comité institutionnel de contrôle (CIC), et à cause de la pratique d'installation des souvenirs positifs avec des mouvements bilatéraux dans le développement du lieu sûr et des ressources au cours de l'EMDR clinique.

\section{Méthodes}

\section{Participants}

Le groupe de participants était composé de 30 jeunes femmes droitières, étudiantes en premier cycle dans une université du sud-ouest des États-Unis, recrutées comme volontaires non cliniques parmi les sujets du département de psychologie. Leur âge moyen était de 19,13 ans $(\sigma=2,56)$ et il n'y avait pas de différence d'âge significative parmi les trois conditions de traitement $(F=0,854 ; p=0,437)$. Aucune des participantes n'était enceinte, aucune ne présentait d'antécédent de blessure à la tête, d'inconscience, d'épilepsie, de douleur chronique, d'antécédents psychiatriques, d'ESPT, ni de neuropathie. Neuf d'entre elles ont déclaré prendre des contraceptifs, deux autres des médicaments non précisés ; l'usage de médicaments était réparti de manière égale dans les trois conditions de traitement $\left(\chi^{2}=0,5 ; p>0,05\right)$. L'usage de drogue était minimal et occasionnel : une participante a rapporté l'usage de marijuana, cinq participantes une consommation d'alcool, trois l'usage d'antidouleurs, une l'usage de stimulants, et trois l'usage d'autres drogues; aucune n'a mentionné l'usage d'amphétamines, de cocaïne, de benzodiazépines, de tranquillisants 
ou d'ecstasy. Chaque participante était assignée de manière aléatoire à l'une des trois conditions de traitement dans ce dispositif intergroupe. Toutes les participantes ont reçu des crédits de cours pour leur participation à cette étude, et l'étude a été approuvée par le CIC de la Northern Arizona University (NAU).

\section{Instruments}

Avant la partie EEG de l'étude, chaque participante a rempli un formulaire d'information démographique contenant des informations d'identification pertinentes : l'âge, le sexe, le statut par rapport à la grossesse, la préférence latérale manuelle, l'incidence des maladies neurologiques susceptibles d'influencer l'enregistrement EEG, les prescriptions médicamenteuses et l'usage récréatif de drogue. En outre, chaque participante a rempli le Edinburgh Handedness Inventory (Oldfield, 1971 ; questionnaire de latéralité manuelle) afin de s'assurer de sa préférence manuelle droite. On a utilisé une échelle visuelle analogue (EVA) 1-10 $(10=$ très fort $)$ afin d'enregistrer la force et la vivacité des souvenirs au départ et après chaque ensemble de stimulus pour chaque condition.

Les conditions de contrôle de la stimulation visuelle consistaient en (a) un point noir statique de 3 pouces $(7,62 \mathrm{~cm})$ de diamètre, condition dite de fixation des yeux (FY), sélectionnée pour contrôler les effets de la stimulation alternée en général et (b) un point alternant entre le vert et le rouge, également de 3 pouces de diamètre, qui changeait de couleur toutes les 500 millisecondes, condition dite du point clignotant, calquée sur la condition de contrôle rapportée dans l'expérience 2 de Christman et coll. (2004). Les deux conditions étaient présentées sur le moniteur 15 pouces d'un ordinateur portable positionné directement en face du sujet, au niveau des yeux et à une distance de 30 pouces $(76,2 \mathrm{~cm})$. Afin d'assurer une cohérence aussi grande que possible, à la fois avec le protocole EMDR et entre les participantes, on a pratiqué les stimulations visuelles bilatérales avec un appareil EyeScan 2000S Light Bar (1994, NeuroTek Corporation, Wheat Ridge, Colorado) conçu pour une utilisation clinique en EMDR. Les allers-retours ont été réglés sur la barre lumineuse à un balayage gauche-droite ou droite-gauche toutes les 500 millisecondes, produisant donc deux mouvements oculaires par seconde, pendant 24 secondes. La barre lumineuse était positionnée au niveau du regard, à approximativement 14 pouces $(35,56 \mathrm{~cm})$ du sujet.

Les informations de l'EEG ont été enregistrées sur un système d'enregistrement EEG Lexicor NRS-24C (1989, Lexicor Medical Technology, Inc., Boulder,
Colorado) ayant un taux d'échantillonnage digital de $512 \mathrm{~Hz}$, un filtre anticrénelage passe-bas à $128 \mathrm{~Hz}$, et un filtre fixe passe-haut à $0,5 \mathrm{~Hz}$. Le Lexicor NRS-24C utilisait une unité d'acquisition Neurosearch-24 contenant 24 canaux de préamplificateurs différentiels avec des amplificateurs/transformateurs d'isolation, des convertisseurs analogique/digital (A/D) et des isolateurs optiques pour la protection des participantes. On a eu recours au logiciel d'enregistrement et d'analyse des EEG Resident Neurosearch-24 V4.1E pour enregistrer les données EEG brutes en fichiers d'événement pour chaque condition de traitement. Les données EEG sur 19 canaux ont été collectées avec un taux d'échantillonnage de conversion $\mathrm{A} / \mathrm{D}$ réglé à $256 \mathrm{~Hz}$, des filtres passe-haut et passe-bas réglés respectivement à $0,5 \mathrm{~Hz}$ et $60 \mathrm{~Hz}$, et un filtre coupe-bande réglé à $60 \mathrm{~Hz}$. Nous avons suivi le système international $10-20$ pour le placement des électrodes EEG pour le positionnement des 19 électrodes $\mathrm{Ag} / \mathrm{AgCl}$ monopodes sur le cuir chevelu des participantes en utilisant l'Electro-Cap System (1983, Electro-Cap International, Inc., Eaton, Ohio) avec des électrodes de référence interauriculaires mathématiquement reliées. Les impédances des électrodes ont été réglées à moins de $5 \mathrm{kohms}$ et à $1 \mathrm{kohm}$ les unes des autres. Les données ont été analysées et artéfactées avec les logiciels d'analyse et de traitement des données Nova Tech EEG EureKa! et MHyT (2000, Nova Tech EEG, Inc., Mesa, Arizona). Les données EEG brutes étaient artéfactées deux fois par deux artéfacteurs formés et indépendants ignorant les conditions de traitement et utilisant des critères écrits rigoureux et précin Un logiciel d'analyse des EEG a été utilisé pour produire des transformations de Fourier rapides (TFR) et des analyses de cohérence et de densité spectrale de puissance des données brutes, et un logiciel de neuro-imagerie LORETA (LORETA : Low Resolution Electromagnetic Tomographic Analysis, Zurich, Suisse) pour produire une imagerie topographique et une localisation corticale des effets du traitement. L'analyse TFR a recouru à l'atténuation du domaine temporel de Hamming, au lissage du domaine fréquentiel de Blackman, un facteur de progression de 8 pour les fenêtres TFR en chevauchement, et un filtre mobile moyen de lissage de 3 . Dans ces analyses, nous avons examiné 10 bandes de fréquence EEG : delta (1-3,99 Hz), thêta $(4-7,99 \mathrm{~Hz})$, thêta basse $(4-5,99 \mathrm{~Hz})$, thêta haute $(6-7,99 \mathrm{~Hz})$, alpha $(8-11,99 \mathrm{~Hz})$, alpha basse $(8-9,99 \mathrm{~Hz})$, alpha haute $(10-11,99 \mathrm{~Hz})$, bêta $(12-30 \mathrm{~Hz})$, bêta basse $(12-19,99 \mathrm{~Hz})$ et bêta haute $(20-30 \mathrm{~Hz})$.

Toutes les données ont été enregistrées dans une cabine de recherche insonorisée, les participantes 
étant assises confortablement dans un siège inclinable. Un miroir était positionné sur le mur opposé et en oblique de manière que le chercheur puisse observer la présence des mouvements oculaires et établir si les yeux de la participante étaient ouverts ou fermés, sans que le sujet voie son reflet dans le miroir. Toutes les instructions ont été standardisées et préenregistrées sur des CD séparés pour chaque condition.

\section{Procédure}

Chacune des participantes s'est vu attribuer de manière aléatoire des rendez-vous EEG et, à leur arrivée, on leur a fait remplir le formulaire obligatoire de consentement éclairé et les questionnaires cités ci-dessus pendant qu'on ajustait l'Electro-Cap sur leur cuir chevelu, qu'on le calibrait au système d'enregistrement EEG et qu'on établissait des tracés EEG propres. Après avoir confortablement installé les participantes et configuré le stimulus visuel, on lançait le CD correspondant au stimulus choisi. L'expérimentateur restait présent pendant la séance pour manipuler l'équipement EEG et surveiller les mouvements oculaires.

Préalablement à la présentation de la condition du stimulus visuel, on enregistrait 5 minutes de tracé EEG de référence pendant laquelle on demandait à la participante de fermer les yeux et de faire le vide dans son esprit, puis " de prendre en compte n'importe quelle pensée, émotion ou expérience qui se manifesterait en elle ». Après cet enregistrement de référence, les participantes étaient invitées à penser à un souvenir épisodique de leur enfance suscitant chez elles des émotions très positives, de signaler à l'examinateur quand elles en avaient trouvé un, et de le lui décrire brièvement et d'en évaluer la force et la vivacité sur l'échelle EVA. On leur demandait ensuite de se concentrer sur le stimulus visuel (le point noir statique, le point clignotant rouge/vert ou le point bilatéral se déplaçant sur la barre lumineuse) pendant qu'elles se concentraient sur leur souvenir épisodique positif pendant 24 secondes. Pendant la condition MOB, on demandait aux participantes de bouger seulement les yeux d'un côté à l'autre, et non leur tête, l'expérimentateur vérifiant grâce au miroir que cette instruction était bien respectée. Après la présentation de la condition du stimulus visuel, on demandait aux participantes de fermer les yeux, de faire le vide dans leur esprit, et de se concentrer sur " n'importe quelle pensée, émotion ou expérience qui se manifesterait » pendant qu'on enregistrait une minute d'EEG. À la fin de cette période d'une minute d'enregistrement, on leur demandait de rapporter à nouveau « la force et la vivacité » du souvenir sur l'échelle EVA de 1 à 10 . Cette séquence de stimulation visuelle, suivie du vidage de l'esprit, puis d'une concentration sur le souvenir au cours de laquelle on enregistrait une minute d'EEG et recueillait la force et la vivacité du souvenir, était répétée cinq fois pendant cinq minutes d'EEG pendant la concentration sur le souvenir épisodique positif, qui suivait elle-même la présentation du stimulus visuel. Après cette séquence d'enregistrements, on débriefait les participantes, on leur ôtait l'Electro-Cap, on leur attribuait leurs crédits et on leur permettait de partir.

\section{Conception et analyse}

Après l'enregistrement de référence d'une durée de cinq minutes où le sujet ferme les yeux et l'enregistrement post stimulus EEG d'une durée de cinq minutes et où le sujet ferme également les yeux, nous avons artéfacté les données et les avons soumises à l'analyse TFR. Le nombre moyen d'époques de 1 seconde sans artéfact, par participante, utilisé dans les analyses de cohérence était de $228,10(\sigma=39,83)$ ou une moyenne de 3,80 minutes $(\sigma=0,66)$ de données EEG sans artéfact pour chaque participante, pour les analyses de référence ou post-stimulus, séparément. Un des résultats produits par le logiciel EureKa! est que les valeurs de cohérence de phase entre toutes les paires possibles d'électrodes pour chaque fréquence EEG prévue, sont générées comme des fonctions de densité spectrales croisées, normalisées par des spectres de densité de puissance individualisés, présentées sous forme de matrice carrée de corrélation pour chaque fréquence. Les analyses de cohérence de phase ont utilisé la formule suivante (Nunez \& Srinivasan, 2006) :

$$
\text { Cohérence }(f)=\frac{\mid \text { Croisé }- \text { Spectre }\left.(f) \mathrm{XY}\right|^{2}}{(\text { Autospectre }[f][\mathrm{X}])(\text { Autospectre }[f][\mathrm{Y}])}
$$

Étant donné l'adaptabilité de ce positionnement pour un nombre relativement petit de groupes d'électrodes, les emplacements de référence pour les calculs de cohérence ont été maintenus sous forme d'électrodes interauriculaires mathématiquement reliées (Nunez \& Srinivasan, 2006 ; Thatcher, Biver $\&$ North, 2004).

À partir de cette matrice de carrés de corrélations, nous avons obtenu les valeurs de cohérence pour les paires d'électrodes concernées. Par rapport à la CIh et afin de simplifier l'analyse par de larges zones fonctionnelles, nous avons sélectionné les paires d'électrodes homologues dans chaque hémisphère, regroupées ainsi : région frontale (Fp1-Fp2, F3-F4, 
F7-F8), centrale (C3-C4), pariétale (P3-P4), temporale (T3-T4, T5-T6) et occipitale (O1-O2). Pour les régions frontale et temporale, on a établi la moyenne, au sein de chaque groupe d'électrodes, des valeurs de cohérence pour chaque paire d'électrodes, afin de donner cinq valeurs de cohérence EEG régionales pour chaque bande de fréquence (voir figure 1). Une analyse exploratoire supplémentaire a examiné la cohérence interhémisphérique afin d'étudier si une des conditions était susceptible d'accroître la coordination de réseaux neuronaux dans les hémisphères. Pour chaque fréquence EEG, frontal gauche ( $\mathrm{Fz}-\mathrm{Fp} 1$, $\mathrm{Fz}-\mathrm{F} 3, \mathrm{Fz}-\mathrm{F} 7)$ et frontal droit $(\mathrm{Fz}-\mathrm{Fp} 2, \mathrm{Fz}-\mathrm{F} 4, \mathrm{Fz}-$ $\mathrm{F} 8)$, central gauche $(\mathrm{Cz}-\mathrm{C} 3, \mathrm{Cz}-\mathrm{T} 3)$ et central droit $(\mathrm{Cz}-\mathrm{C} 4, \mathrm{Cz}-\mathrm{T} 4)$, pariétal gauche (Pz-P3, Pz-T5) et pariétal droit $(\mathrm{Pz}-\mathrm{P} 4, \mathrm{Pz}-\mathrm{T} 6)$, occipital gauche $(\mathrm{Pz}-$ $\mathrm{O} 1)$ et occipital droit $(\mathrm{Pz}-\mathrm{P} 2)$, nous avons comparé les groupes de cohérence intrahémisphérique par région. Afin de réduire le nombre d'analyses séparées, nous avons établi la moyenne des valeurs de cohérence dans chaque groupe de régions (frontal, central pariétal). Ces ensembles de données inter- et intrahémisphériques ont été ensuite étudiés pour déterminer la normalité et l'homogénéité des hypothèses de variance et nous avons constaté qu'ils correspondaient bien à l'hypothèse de la nécessité d'analyses paramétriques supplémentaires. Les valeurs de cohérence pour chaque région du cerveau ont été ensuite examinées au sein de chaque condition et pour chacune des 10 bandes de fréquence EEG, orthogonalement, selon la fréquence et l'hémisphère, par des analyses intergroupes de la covariance (ANCOVA), avec pour covariable les valeurs de base respectives. Dans la mesure où il s'agissait d'une étude portant sur un nombre restreint de sujets, avec des comparaisons planifiées, et qu'il n'y avait pas plus de comparaisons que de degrés de liberté pour l'effet, il n’a pas été nécessaire de procéder à un ajustement pour une inflation du FWER (familywise error rate) (Tabachnik \& Fidell, 2013). Le seuil de signification alpha a été réglé à 0,05 .

De plus, afin de mieux localiser régions du cerveau potentiellement affectées par la stimulation visuelle au cours de la visualisation des souvenirs épisodiques positifs, nous avons utilisé le logiciel d'analyse tomographique électromagnétique à résolution basse (LORETA). LORETA est un logiciel compagnon d'imagerie cérébrale tridimensionnelle, compagnon des analyses EEG actuelles permettant la localisation de potentiels de source corticale profonde pour les signaux EEG de surface enregistrés (Pascual-Marqui, Esslen, Kochi \& Lehmann, 2002). Les algorithmes LORETA calculent un espace tridimensionnel de solutions inverses de matière grise corticale et des hippocampes projetés sur un atlas probabiliste de Talairach partagé en 2394 unités volumétriques de $7 \mathrm{~mm} 3$, ou voxels. La nomenclature anatomique de Brodmann peut être utilisée pour les régions qui nous intéressent en utilisant le modèle réaliste de la tête du Montreal Neurological Institute. Pour cette étude, les analyses LORETA ont été menées sur la transformation logarithmique naturelle des résultats TFR relatifs du spectre de puissance pour chaque fréquence identifiée et les voxels corticaux statistiquement significatifs pertinents ont été rapportés.

\section{Résultats}

\section{Effets sur la force et la vivacité du souvenir}

La figure 2 présente graphiquement les changements dans les évaluations de la force et de la vivacité des souvenirs pour chaque condition, en partant de la base de référence et pour chacun des cinq tests de stimulation visuelle. Les résultats des analyses des mesures répétées de variance (ANOVA) montrent un effet principal important pour le temps $(F[5,23]=7,80 ; p<0,0001$; $\left.\eta^{2}=0,63\right)$ mais pas pour la condition $(F[2,27]=0,59$; $\left.p=0,56 ; \eta^{2}=0,04\right)$ et aucune interaction significative $\left(F[10,46]=0,54 ; p=0,86 ; \eta^{2}=0,10\right)$, indiquant une augmentation de la force et de la vivacité du souvenir positif au fil du temps pour les trois conditions, et aucune différence parmi les conditions à aucun point de mesure, y compris au début. Un contrôle de ces graphiques, cependant, révèle un pattern de réponses différent pour la condition dite MOB et pour les deux autres conditions de contrôle, ces dernières atteignant le plateau au troisième test, le souvenir augmentant plutôt régulièrement parmi tous les tests sauf un pour la condition MOB. Des comparaisons du test $t$ entre les tests successifs vont dans le sens de cette différence de pattern visuel, avec une augmentation importante pour la condition FY uniquement entre les tests 2 et 3 $(t[9]=4,00 ; p=0,002)$ et pour la condition du point clignotant seulement entre les tests 1 et $2(t[9]=1,81$; $p=0,05)$, mais pour la condition MOB, entre les tests 1 et $2(t[9]=1,96 ; p=0,04)$, entre les tests 2 et 3 $(t[9]=1,81 ; p=0,05)$ et entre les tests 4 et $5(t[9]=$ $1,81 ; p=0,05)$.

\section{Effets de cohérence interhémisphérique}

Les analyses interhémisphériques ANCOVA n'ont obtenu aucun effet principal important dans les conditions (tous les $p>0,05$ ). De simples comparaisons d'effets entre chaque condition pour chaque fréquence n'ont révélé qu'un seul effet de condition statistiquement significatif : la cohérence de la condition dite 


\section{Memory Strength Across Trials}

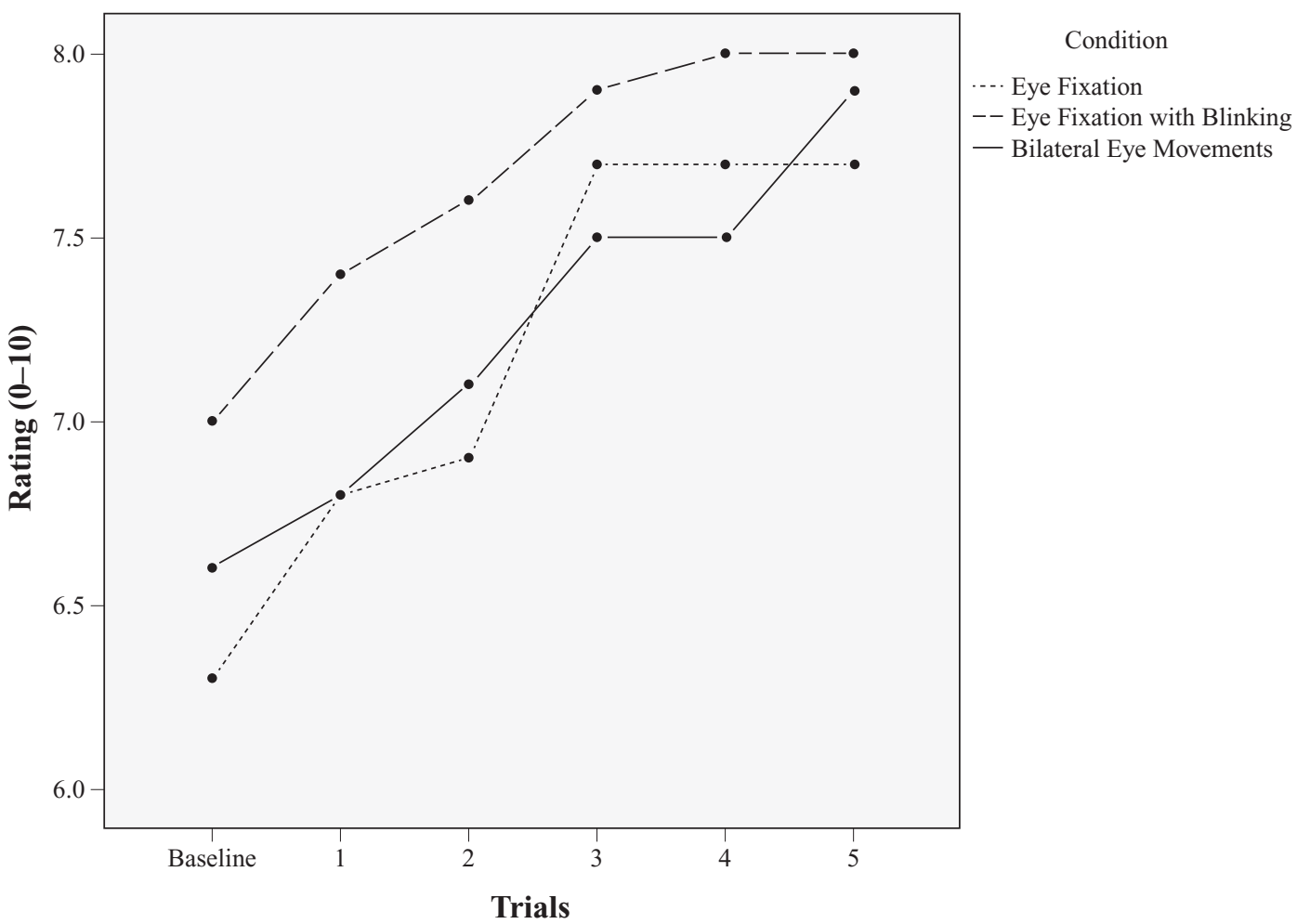

FIGURE 2. Évaluation de la force et de la vivacité du souvenir au fil des tests de mesure EEG pour chaque condition.

Note. Memory strength across trials $=$ force du souvenir au fil des tests $;$ Rating $=$ notes $;$ Baseline $=$ départ $;$ Condition $=$ condition $;$ Eye Fixation $=$ fixation des yeux ; Eye Fixation with blinking $=$ fixation des yeux avec clignotement $;$ Bilateral Eye Movements $=$ mouvements oculaires bilatéraux.

du point clignotant était plus élevée que celle de la condition YF pour la bande thêta centrale $(p=0,028)$. On a constaté des tendances statistiques où la condition MOB montrait une cohérence plus élevée que la condition YF pour la bande delta frontale $(p=0,081)$ et que la condition du point clignotant pour la bande alpha basse occipitale $(p=0,051)$ ainsi qu'une tendance statistique de cohérence plus élevée pour la condition du point clignotant que pour les MOB pour la bande alpha centrale $(p=0,054)$, pour la bande centrale $(p=0,066)$ et pour la bande bêta pariétale $(p=0,096)$. Aucune condition pour aucune région ou fréquence n'a atteint la signification statistique ou un statut de tendance.

\section{Effets de cohérence intrahémisphérique}

Les analyses ANCOVA intrahémisphériques ont montré plusieurs effets principaux importants et tendanciels liés à la condition. Pour la delta frontale droite, nous avons obtenu une tendance statistique pour la condition (ANCOVA $F[2,26]=3,161 ; p=$ 0,$\left.059 ; \eta^{2}=0,196\right)$. Les comparaisons planifiées d'effets simples ont montré que la condition MOB avait une cohérence significativement plus élevée que la condition du point clignotant $(p=0,028)$ et une tendance à une cohérence plus élevée par rapport à la condition YF $(p=0,055)$. Des comparaisons des changements par des tests $t$, avant et après exposition à chaque condition, ont révélé une augmentation significative de la cohérence $\mathrm{MOB}$ pour la bande delta frontale droite $(t[9]=-2,50 ; p=0,017)$ mais pas de changement significatif pour les conditions YF $(t[9]=-0,25 ; p=0,43)$ ou du point clignotant $(t[9]=$ $-0,33 ; p=0,38)$. Fait important, nous n'avons découvert aucun effet important ni de tendance d'effet pour aucune des trois conditions pour la bande delta frontale gauche.

Pour la bande bêta frontale droite, nous avons observé une tendance statistique pour la condition (ANCOVA $F[2,26]=3,092 ; p=0,062, \eta^{2}=$ $0,192)$. Les comparaisons planifiées ont montré que la condition $\mathrm{MOB}$ avait une cohérence significativement plus élevée que la condition du point clignotant $(p=0,022)$. Afin de démêler les contributions des bandes de fréquence bêta basse et bêta haute pour cet effet bêta, les analyses de la bande bêta basse frontale droite (ANCOVA $F[2,26]=2,340$; 
$\left.p=0,019 ; \eta^{2}=0,263\right)$ ont révélé que les conditions $\operatorname{MOB}(p=0,008)$ et YF $(p=0,034)$ avaient une cohérence significativement plus élevée que la condition du point clignotant et que la bande bêta haute frontale droite de la condition MOB (ANCOVA $F[2,26]=$ 2,$\left.340 ; p=0,116 ; \eta^{2}=0,153\right)$ était significativement plus élevée que la condition du point clignotant $(p$ $=0,044)$. Pour la bande bêta basse frontale gauche (ANCOVA $F[2,26]=2,315 ; p=0,119 ; \eta^{2}=0,151$ ), les analyses ont montré que la cohérence pour la condition MOB était significativement plus élevée que celle de la condition du point clignotant $(p=$ 0,042). Les figures 3-5 montrent de façon descriptive les changements de cohérence dans les bandes bêta basse frontale droite, bêta haute frontale droite et bêta basse frontale gauche, avant et après exposition à chaque condition, révélant des augmentations de la cohérence pour la condition $\mathrm{MOB}$, des diminutions de la cohérence pour la condition du point clignotant, et de légères diminutions ou aucun changement notable de la cohérence dans la condition YF. Les comparaisons par des tests $t$ de ces changements, avant et après exposition à chacune des conditions, sont présentées dans le tableau 1, montrant une augmentation importante de la cohérence MOB pour la bande bêta basse frontale gauche et une tendance statistique vers une augmentation pour la bande bêta basse frontale droite, avec une diminution importante correspondante de la cohérence du point clignotant pour la bande bêta basse frontale droite et une tendance à la diminution pour la bande bêta haute frontale droite, et aucun changement ou tendance au changement significatifs de la cohérence pour la condition YF. Aucune autre région corticale et aucune fréquence n'ont révélé d'effets significatifs des MOB, par rapport aux deux conditions de contrôle, sur la cohérence EEG intra-hémisphérique 2

\section{Localisation des effets des mouvements oculaires bilatéraux}

Les algorithmes de localisation de source corticale de la neuro-imagerie LORETA ont été appliqués aux données EEG des spectres de puissance relative

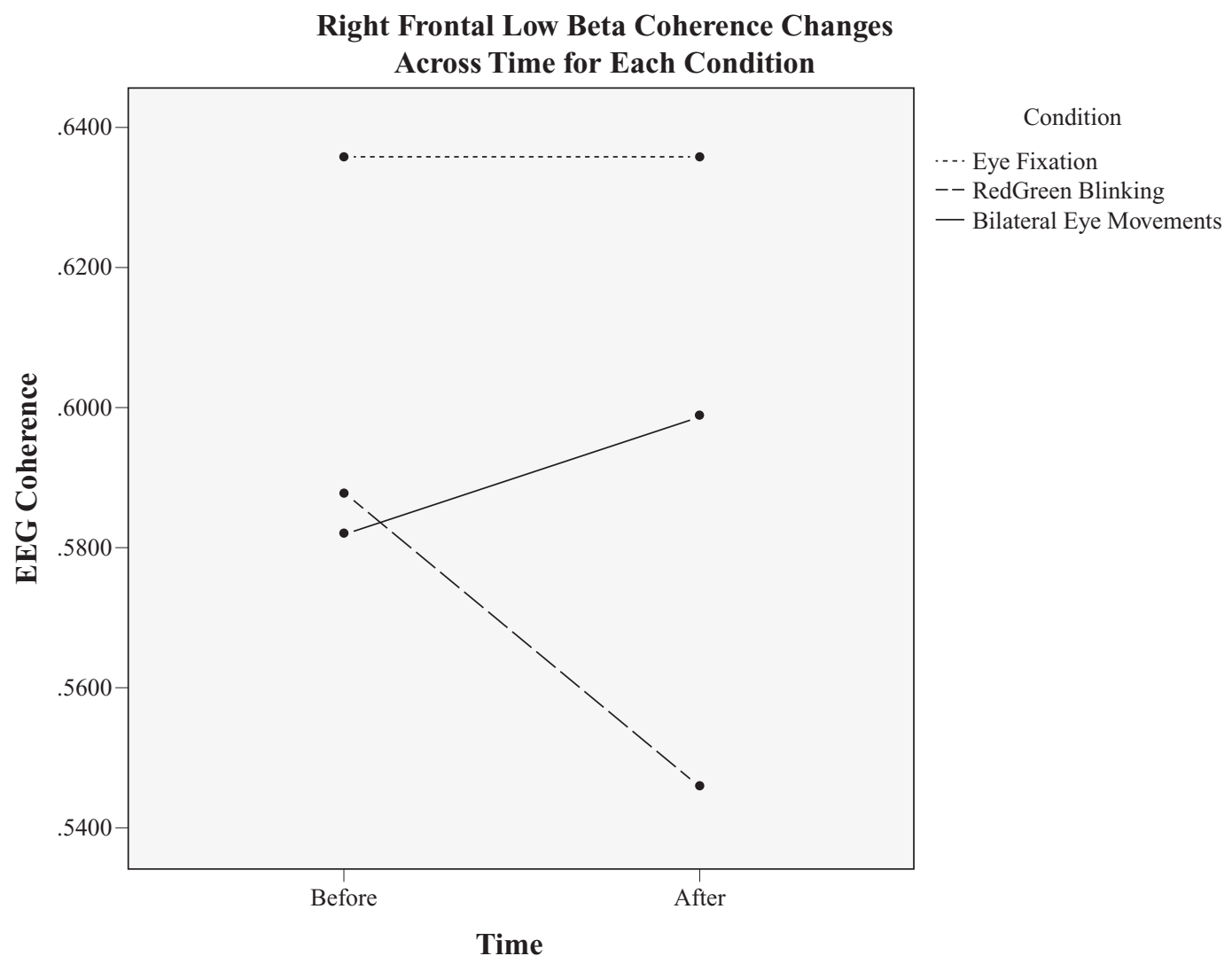

FIGURE 3. Changements de cohérence dans les ondes bêta basses frontales droites en fonction du temps pour chaque condition.

Note. Right Frontal Low Beta Coherence Changes Across Time for Each Condition = changements de cohérence dans les ondes bêta basses frontales droites en fonction du temps pour chaque condition ; EEG Coherence = cohérence EEG ; Condition $=$ condition ; Eye Fixation $=$ fixation des yeux $;$ RedGreen Blinking = Clignotement rouge vert ; Bilateral Eye Movements $=$ mouvements oculaires bilatéraux. 


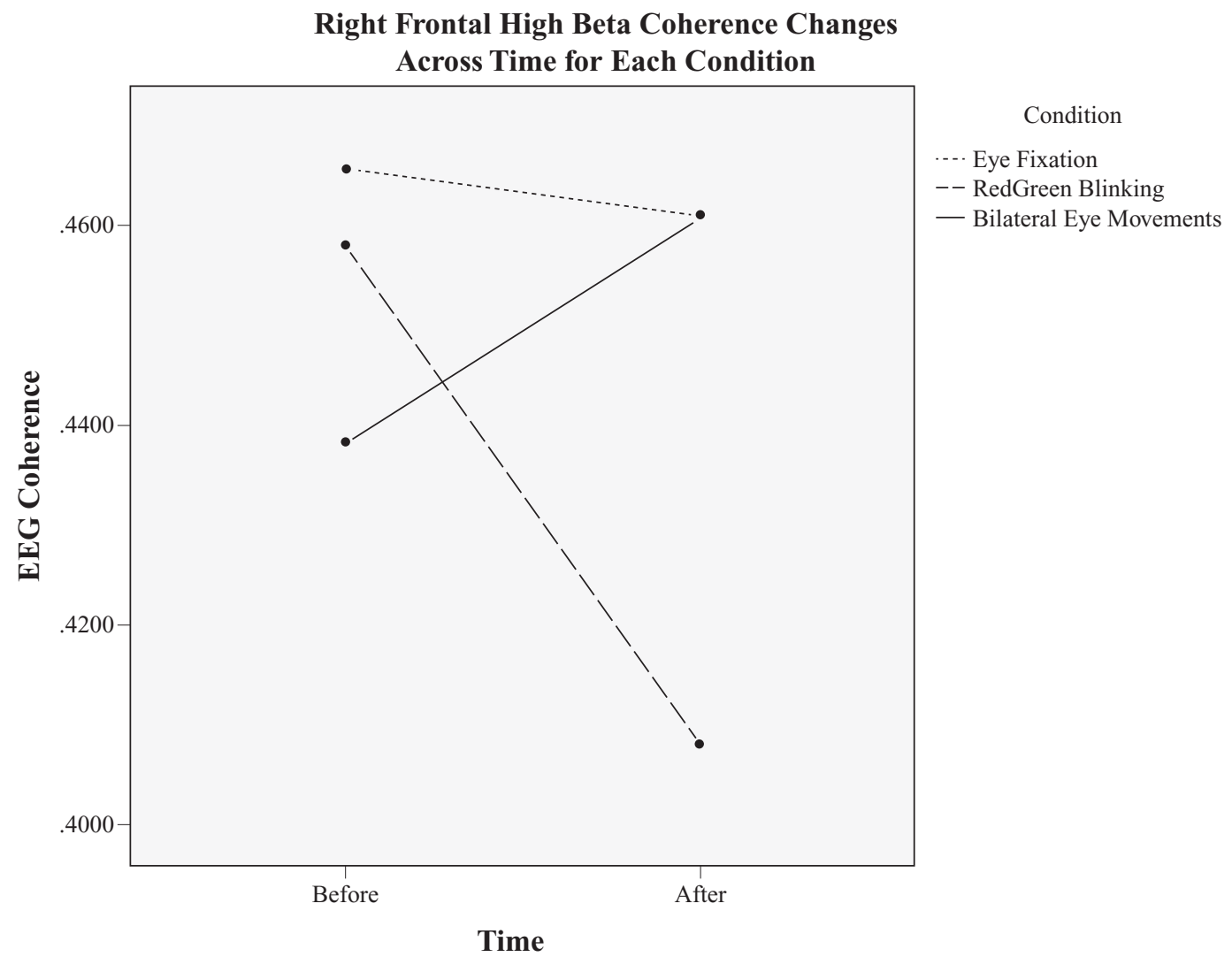

FIGURE 4. Changements de cohérence dans les ondes bêta hautes frontales droites en fonction du temps pour chaque condition.

Note. Right Frontal High Beta Coherence Changes Across Time for Each Condition = Changements de cohérence dans les ondes bêta frontales droites en fonction du temps pour chaque condition ; EEG Coherence = cohérence EEG ; Condition $=$ condition $;$ Eye fixation $=$ fixation des yeux $;$ RedGreen Blinking $=$ clignotement rouge vert ; Bilateral Eye Movements $=$ mouvements oculaires bilatéraux.

tirées des cinq époques d'enregistrement combinées d'une minute, à la suite de la mise en œuvre de chacune des trois conditions. Ce processus d'imagerie produit des images virtuelles à résonance magnétique (IRM) des régions corticales significativement actives au cours de chaque condition. Dans cette étude, nous nous intéresserons seulement aux images LORETA fournies pour la condition MOB. La figure 6 montre les images supérieure, postérieure, latérale gauche, inférieure, antérieure et latérale droite de l'activation bêta basse, après les MOB. Les zones ombrées reflètent l'activation des aires de Brodmann 10 et 11 dans les gyrus supérieur droit et frontal moyen.

\section{Discussion}

Les résultats de notre étude ne soutiennent guère le modèle CIh des effets thérapeutiques de l'EMDR. Les MOB n'ont produit que des augmentations tendancielles de cohérence entre les hémisphères, comparativement aux conditions YF et des points lumineux rouges/verts alternés, et ces tendances allaient vers des augmentations de cohérence des ondes lentes, delta et alpha basse, c'est-à-dire des fréquences électrocorticales qui ne sont généralement pas associées au traitement de l'information. Klimesch et coll. (Klimesch, 1999 ; Klimesch, Sauseng \& Hanslmayr, 2007) ont suggéré que la synchronisation alpha pouvait refléter un processus inhibiteur dans la préparation de la récupération des souvenirs et des processus cognitifs subséquents. Il est tentant d'imaginer que cette tendance alpha basse, se produisant dans les régions occipitales, puisse suggérer le commencement d'une activité cohérente dans les zones de traitement impliquées dans la récupération des composants visuels des souvenirs positifs évoqués. Cette hypothèse devra faire l'objet d'études plus larges pour une meilleure validation.

Notre étude soutient cependant le modèle de cohérence intrahémisphérique pour les effets de l'EMDR. Pour les fréquences EEG plus élevées et les régions corticales frontales, reconnues pour être impliquées dans le traitement d'ordre supérieur des informations, cette étude permet d'observer que les 
Left Frontal Low Beta Coherence Changes

Across Time for Each Condition

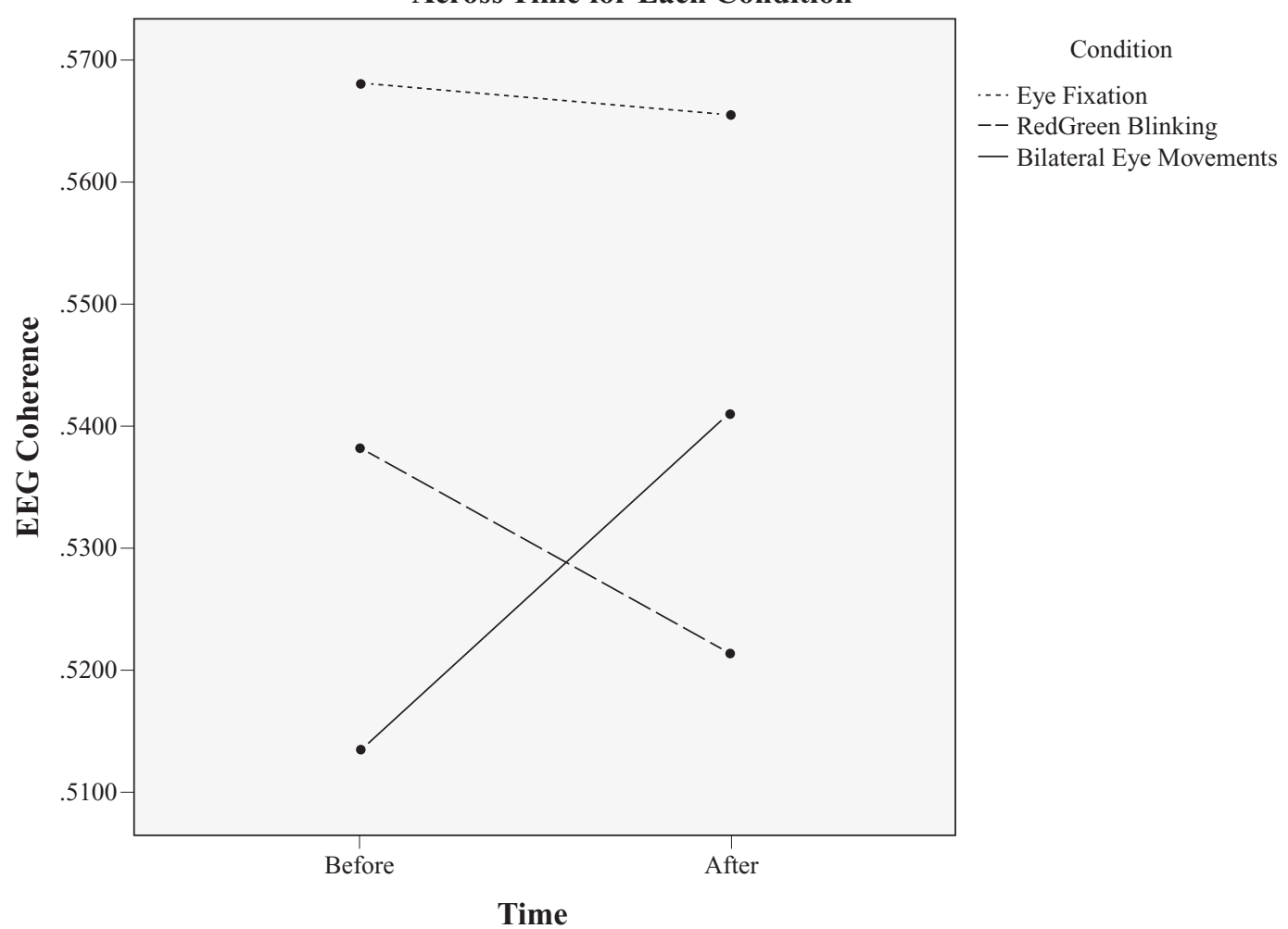

FIGURE 5. Changements de cohérence EEG dans les ondes bêta basses frontales gauches en fonction du temps pour chaque condition.

Note. Left Frontal Low Beta Coherence Changes Across Time for Each Condition = changements de cohérence EEG dans les ondes bêta basses frontales gauches en fonction du temps pour chaque condition ; EEG Coherence = cohérence EEG ; Condition $=$ condition $;$ Eye Fixation $=$ fixation des yeux $;$ RedGreen Blinking $=$ clignotement rouge vert ; Bilateral Eye Movements $=$ mouvements oculaires bilatéraux.

MOB sont associés à une amélioration de la cohérence EEG. Nous avons en particulier constaté que les fréquences bêta basses frontales gauches et droites et la fréquence bêta haute frontale droite montrent une augmentation de la cohérence après la stimulation MOB, au cours de la visualisation d'un souvenir émotionnel positif, alors que nous avons constaté des diminutions de la cohérence pour la condition $\mathrm{du}$ point clignotant et aucun changement pour la condition YF. De plus, parmi les trois conditions, ce pattern d'effets différentiels a montré une cohérence à travers d'autres régions corticales pour la fréquence bêta, même si ces observations n'approchent pas de niveaux tendanciels ou importants. Et ces changements des EEG bêta frontales étaient associés avec un pattern d'augmentation unique et plus continu de la force et de la vivacité du souvenir épisodique positif ciblé.

Les résultats de la neuro-imagerie LORETA étaient également en cohérence avec une activation préfrontale droite dans la bande de fréquence bêta basse. De plus en plus d'études indiquent l'implication du cortex préfrontal droit dans les états affectifs négatifs (Davidson, 1995, 2002) et dans la réponse empathique (Tullet, Harmon-Jones \& Inzlicht, 2012). Il est intéressant de noter que notre étude a constaté une augmentation de la cohérence des ondes bêta basse et haute, et une augmentation du spectre de puissance de l'onde bêta basse dans le cortex préfrontal droit au cours de la visualisation d'états émotionnels positifs. Ces résultats sembleraient en contradiction avec ceux de Davidson; cependant, notre étude a également constaté une augmentation de la cohérence dans le cortex préfrontal gauche, une région considérée comme étant aussi impliquée dans les états émotionnels positifs (Davidson, 2002). Nous ne pouvons qu'émettre l'hypothèse, à l'heure actuelle, que la tâche cognitive de visualisation des souvenirs émotionnels positifs, pendant la stimulation bilatérale, implique l'activation équilibrée des régions droite et gauche du traitement émotionnel, bien qu'il ne s'agisse 
TABLEAU 1. Résultats de test $-t$ d'échantillons appariés (référence du post-traitement) pour les effets de cohérence intrahémisphérique pour les ondes bêta frontales

\begin{tabular}{|c|c|c|c|}
\hline Condition/Hémisphère/Fréquence & Erreur standard de la mesure (ES) & Valeur de $t$ & Valeur de $p$ \\
\hline \multicolumn{4}{|l|}{ Yeux fixes } \\
\hline Bêta frontale gauche & $0,016(0,017)$ & 0,922 & 0,191 \\
\hline Bêta basse frontale gauche & $0,003(0,013)$ & 0,194 & 0,426 \\
\hline Bêta haute frontale gauche & $0,018(0,015)$ & 1,196 & 0,131 \\
\hline Bêta frontale droite & $0,006(0,021)$ & 0,274 & 0,396 \\
\hline Bêta basse frontale droite & $-0,000(0,014)$ & $-0,017$ & 0,494 \\
\hline Bêta haute frontale droite & $0,005(0,018)$ & 0,252 & 0,404 \\
\hline \multicolumn{4}{|l|}{ Point clignotant rouge/vert } \\
\hline Bêta frontale gauche & $0,018(0,016)$ & 1,137 & 0,143 \\
\hline Bêta basse frontale gauche & $0,017(0,014)$ & 1,197 & 0,131 \\
\hline Bêta haute frontale gauche & $0,022(0,021)$ & 1,012 & 0,169 \\
\hline Bêta frontale droite & $0,048(0,024)$ & 1,999 & $0,039^{\star}$ \\
\hline Bêta basse frontale droite & $0,042(0,017)$ & 2,437 & $0,019^{\star}$ \\
\hline Bêta haute frontale droite & $0,050(0,028)$ & 1,770 & 0,055 \\
\hline \multicolumn{4}{|l|}{ Mouvements oculaires bilatéraux } \\
\hline Bêta frontale gauche & $-0,026(0,020)$ & $-1,329$ & 0,108 \\
\hline Bêta basse frontale gauche & $-0,028(0,015)$ & $-1,869$ & $0,047^{\star}$ \\
\hline Bêta haute frontale gauche & $-0,017(0,024)$ & $-0,710$ & 0,248 \\
\hline Bêta frontale droite & $-0,023(0,016)$ & $-1,452$ & 0,090 \\
\hline Bêta basse frontale droite & $-0,017(0,011)$ & $-1,538$ & 0,079 \\
\hline Bêta haute frontale droite & $-0,022(0,022)$ & $-1,001$ & 0,172 \\
\hline
\end{tabular}

Note. Tous les tests sont des tests unilatéraux où $\mathrm{df}=9$. Erreur standard négative de la mesure $\rightarrow$ la cohérence après traitement était plus importante qu'avant.

${ }^{\star} p<0,05$.

pas d'une interconnexion des hémisphères droits et gauches comme l'aurait indiqué une augmentation de la CIh.

Nous avons également constaté une augmentation de la cohérence de la fréquence delta dans le lobe frontal droit pour la condition MOB. Il serait tentant d'interpréter cette augmentation de la cohérence d'onde basse comme un artéfact résiduel de l'activité MOB se produisant avant l'enregistrement EEG. Nous pensons que ce n'est pas le cas, pour deux raisons : premièrement, nous avons artéfacté visuellement, à deux reprises, les fichiers EEG en faisant particulièrement attention au risque d'artéfacts liés aux mouvements oculaires dans les électrodes frontales en utilisant les procédures écrites, les critères et la formation établis au cours de plus de dix ans de recherches dans nos laboratoires. De plus, si cette augmentation de la cohérence delta était causée par les artéfacts $\mathrm{MOB}$, elle se produirait à la fois sur les deux côtés, ce qui n'a pas été le cas dans cette analyse. Nous pensons qu'une autre explication à ce résultat de la cohérence delta est plus défendable. Bien que le rythme EEG delta ait été historiquement associé aux étapes développementales précoces, au sommeil et à certains processus pathologiques, il a été constaté plus récemment qu'il soit lié à la synchronisation du cerveau avec des fonctions autonomes, certains états motivationnels de défense ou fondés sur la récompense, et la détection et l'attention portée aux stimuli marquants (Knyazev, 2012). Étant donné que les participantes de notre étude venaient d'être traitées pour une expérience émotionnelle mnésique positive et, nous l'espérons, gratifiante, il semble plus plausible que cette amélioration de la cohérence delta reflète l'attention portée à cet état émotionnel positif.

Le cortex préfrontal a longtemps été associé aux fonctions exécutives, plus spécifiquement à la 


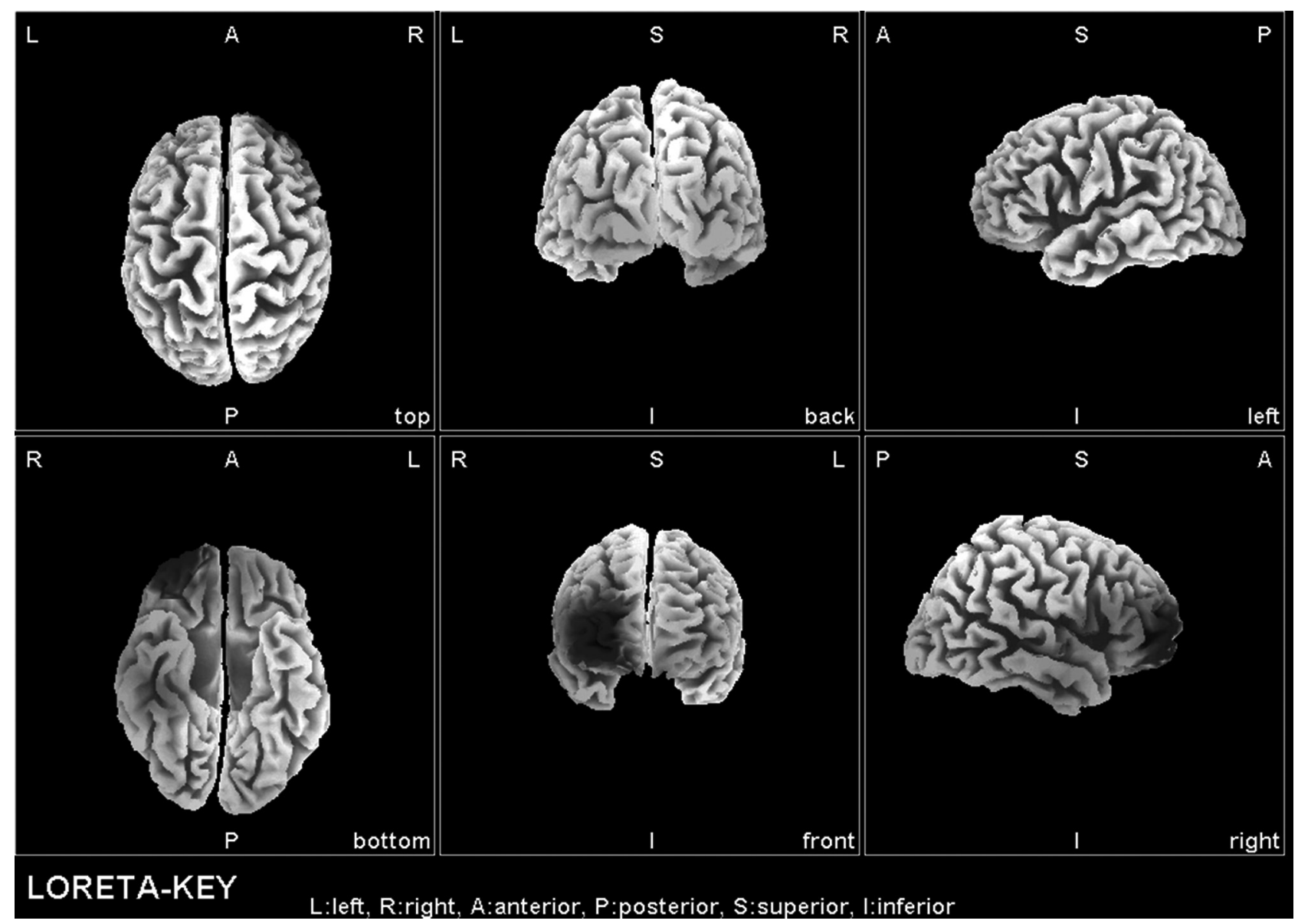

FIGURE 6. Vues orthographiques de neuroimagerie EEG LORETA pour le spectre de puissance bêta basse à la suite de mouvements oculaires pendant la visualisation d'un souvenir émotionnel positif (les zones ombrées représentent une puissance significativement augmentée par rapport aux zones claires).

planification des comportements complexes, la différentiation positif/négatif, la prédiction de résultats, la prise de décision, l'expression de la personnalité et le comportement prosocial - beaucoup des processus impliqués dans la sélection et la visualisation d'un souvenir émotionnel positif. Les aires de Brodmann 10 et 11 , identifiées à partir des résultats de neuroimagerie, sont des subdivisions du cortex préfrontal plus particulièrement impliquées dans la récupération de souvenirs épisodiques, les comportements médiés par la récompense, l'empathie cognitive et l'originalité et la flexibilité cognitive (Ramnani \& Owen, 2004 ; Trans Cranial Technologies, 2012). Le fait que ces régions aient montré une augmentation importante de l'activation et de la cohérence à la suite des MOB, lors du rappel de souvenirs épisodiques positifs, pour la fréquence EEG bêta basse que l'on associe le plus souvent à la concentration et à l'attention, cadre bien avec les exigences de la tâche (Bergmann, 2008). De plus, ces résultats de localisation sont cohérents avec les études de la tomographie d'émission monophotonique assistée par ordinateur (SPECT), qui montrent une augmentation de l'afflux sanguin dans les zones limbiques et du CPF après l'EMDR et pourraient refléter un recouplage des régions amygdale-CCA / CPF, ainsi que nous l'avons décrit plus haut (Pagani et coll. 2013).

Il est important de noter que des études EEG antérieures portant sur les effets de cohérence des MOB n'ont pas suivi ce protocole au cours de la visualisation du souvenir, comme cela se produit dans la thérapie EMDR. Nous pensons qu'il s'agit là d'une omission importante de la part de ces études, dans la mesure où cela a limité leur pertinence par rapport au processus formel de l'EMDR. Notre étude a employé la visualisation de souvenirs positifs lors des MOB. La constellation de résultats est très probablement lourdement influencée par la tâche spécifique utilisée dans notre étude, et ces résultats vont être probablement différents ou inclure d'autres régions pour la visualisation de souvenirs négatifs ou traumatiques. En effet, les résultats de la neuro-imagerie, ainsi 
que la localisation des effets de cohérence corticale, sont apparus très spécifiques aux processus cognitifs impliqués dans cette tâche unique. Il ne serait pas surprenant d'observer une augmentation de la cohérence et de l'activation d'autres régions corticales distinctes ayant des fonctions spécifiques aux tâches menées. Par exemple, nous pourrions nous attendre, lors de la visualisation de souvenirs négatifs ou traumatiques, à voir les mêmes régions mentionnées plus haut être impliquées, avec une activation d'un nombre plus grand de zones frontales médianes, reflétant l'activation des sous-cortex CA et amygdalien. Ces spéculations attendent de nouvelles études sur la cohérence EEG et la neuro-imagerie.

Concernant ces nouvelles études EEG sur les MOB, l'observation des résultats pour la condition du point clignotant nous indique que la condition de contrôle de la fixation visuelle vert/rouge alternée, utilisée dans des études antérieures par Propper et coll. (2007) et par Samar et coll. (2011), a des effets plutôt remarquables sur la cohérence EEG. Dans une analyse EEG post hoc, nous avons constaté que la cohérence de l'onde thêta centrale, pour la condition du point clignotant, était significativement plus élevée que dans la condition YF $(p=0,028)$, et dans une analyse intrahémisphérique similaire, la cohérence du point clignotant était significativement plus élevée que la cohérence YF, pour la delta pariétale droite $(p=0,04)$ et pour la thêta pariétale gauche $(p=0,024)$, avec un pattern tendanciel identique pour les autres sites pariétal et central gauche/droit pour ces fréquences basses. En effet, la condition YF lumineuse vert/rouge alternée semblait augmenter de manière constante la cohérence de fréquence basse dans les régions corticales centrale et pariétale et diminuer la cohérence de fréquence haute dans les régions frontales. Cet effet est en contraste avec une tendance du stimulus MOB à diminuer la cohérence de fréquence basse et d'augmenter la cohérence de fréquence haute dans des régions corticales spécifiques. Étant donné que les fréquences delta et thêta sont traditionnellement associées à la sédation, au sommeil et aux états de transe, et que les fréquences bêta sont associées à la concentration, la vigilance et leurs fonctions associées (Bergmann, 2008 ; Stevens et coll., 2004), le résultat général de cette étude cadre avec l'augmentation de la vigilance, de la concentration et des associations au cours de la stimulation MOB et une désactivation contradictoire du traitement cognitif pendant la condition du point clignotant. Bien que la comparaison de la condition MOB et de celle du point clignotant puisse augmenter la magnitude de l'effet, l'utilisation du point clignotant alternant entre une lumière rouge/verte n'est peut-être pas le meilleur choix pour une condition de comparaison de contrôle et peut avoir une validité externe limitée.

Bien sûr, il est possible que les différences que nous avons obtenues entre la condition MOB et les conditions de contrôle aient un certain rapport avec la présentation des conditions de contrôle sur un écran d'ordinateur et l'utilisation d'une barre lumineuse pour la stimulation bilatérale. Cependant, il nous semble que la barre lumineuse capte et standardise mieux la stimulation MOB dans les dispositifs cliniques que les points apparaissant alternativement sur les deux côtés d'un écran informatique, utilisés dans les études antérieures. Ainsi, la validité de nos résultats nous paraît meilleure, sur le plan externe, que ceux obtenus à partir de points générés par ordinateur. À cet égard, il est à noter qu'une réplication constructive de cette étude utilisant la barre lumineuse pour les trois conditions, actuellement sous analyse, ait obtenu un effet différentiel similaire parmi les trois conditions.

Un résultat non-EEG intéressant de notre étude a concerné la découverte d'une augmentation de la force et de la vivacité du souvenir à la suite des trois conditions, avec un pattern d'augmentation différent pour la condition MOB. Ce résultat est en nette contradiction avec un vaste corpus de recherche dont les constatations vont dans le sens d'une diminution de la vivacité des souvenirs positifs et négatifs à la suite des MOB (Engelhard, Van Uijen \& Van den Hout, 2010 ; Gunter \& Bodner, 2008 ; Hornsveld et coll., 2011 ; Maxfield, Melnyk \& Hayman, 2008 ; Van den Hout, Eidhof, Verboom, Little \& Engelhard, 2013 ; Van den Hout \& Engelhard, 2012 ; Van den Hout, Muris, Salemik \& Kindt, 2001). Ces études ont été conçues de la manière suivante : les participants se rappellent un souvenir, ils évaluent sa vivacité, puis ils se rappellent encore ce souvenir pendant plusieurs séquences de mouvements oculaires ou de fixations, puis, après une période d'attente variable, ils réévaluent la vivacité du souvenir immédiatement après son rappel et la mise en ouvre de la condition. Le plus souvent, les souvenirs semblent moins vivaces après les MOB qu'à la suite de la condition YF seule (Maxfield et coll., 2008 ; Van den Hout et al. 2013). Ce dispositif et ces résultats basiques, appelés « déflation de l'imagination " avaient pour but de soutenir une explication de l'EMDR fondée sur la mémoire de travail : l'EMDR saturerait l'espace visuospatial interne du sujet (Andrade, Kavanagh \& Baddeley, 1997) ou son comité exécutif central (Gunter \& Bodner, 2008) dans la mémoire de travail, en l'obligeant à se centrer simultanément sur le souvenir et sur les mouvements 
oculaires, ce qui produit un brouillage et un affaiblissement subséquent de la vivacité du souvenir.

Cependant, ainsi que nous l'avons rapporté dans notre étude, il s'est avéré que les évaluations de la force et de la vivacité du souvenir montraient une augmentation significative pour toutes les conditions, avec toutefois une augmentation plus marquée pour la condition MOB. Afin d'expliquer cette contradiction avec un corpus de recherche bien établi, il faut comprendre qu'il existe une différence fondamentale entre la conception de notre étude et celles des études antérieures. Dans notre étude, plus cohérente avec la pratique EMDR, les évaluations de la vivacité du souvenir étaient menées après chacune des cinq périodes de traitement séquentiel d'1 minute qui suivaient la mise en œuvre de la condition du stimulus. Dans la conception de recherche classique de la stimulation bilatérale de la mémoire de travail, la vivacité est évaluée immédiatement après la mise en œuvre de la condition, sans aucune période de traitement. En fait, nous n'avons trouvé, dans l'examen de cette littérature sur les mouvements oculaires, qu'une seule étude (Lee \& Drummond, 2008) qui rapportait une évaluation de la vivacité après la phase 4 de la pratique EMDR clinique, l'évocation répétée du souvenir avec stimulation bilatérale, suivie chaque fois par une brève période de traitement, jusqu'à ce que les notes d'unités subjectives de perturbation (échelle SUD) soient ramenées à zéro (Shapiro, 2001). Et ces auteurs n'avaient pas réussi à obtenir de diminution de la vivacité quand les sujets " revivaient " le souvenir, mais ils en obtenaient une lorsqu' on demandait aux sujets de se distancier du souvenir, et cet effet n'apparaissait qu'immédiatement après la désensibilisation et non lors du suivi, une semaine plus tard. Il nous semble que cette différence conceptuelle explique nos résultats apparemment contradictoires.

Cela dit, plutôt que de contester l'hypothèse de la mémoire de travail sur cette contradiction procédurale, nous voudrions proposer une explication alternative aux effets des MOB dans la pratique EMDR. Nous suggérerions que l'EMDR fonctionne dans un processus à deux étapes. Dans la première étape, les souvenirs sont brouillés et diminués via un processus de saturation de la mémoire de travail, bien décrit et appuyé par les contributions de Van den Hout et coll. (2013; Van den Hout \& Engelhard, 2012), Maxfield et coll. (2008), Gunter \& Bodner (2008), Andrade et coll. (1997) et d'autres auteurs. Cependant, cet effet de désensibilisation est suivi, dans la pratique courante de l'EMDR, par une seconde étape de traitement au cours de laquelle des liens associatifs sont formés entre la trace du souvenir brouillé et des expériences mnésiques qui lui sont liées, avec pour résultat une augmentation de la vivacité mnésique d'une réélaboration plus constructive du souvenir originel. Maxfield et coll. ont suggéré la même séquence de détérioration ciblée de la trace du souvenir d'origine suivie d'une augmentation de la vivacité à travers des liens associatifs constructifs subséquents. Nos résultats, montrant une cohérence EEG améliorée avec des souvenirs positifs, soutiennent très timidement cette deuxième étape. Ce traitement en deux étapes peut être facilement testé en élargissant simplement le paradigme actuel de la mémoire de travail afin d'y inclure une réévaluation de la vivacité après une période subséquente de traitement. Si cette séquence d'effets est confirmée par d'autres études de cette nature, elles pourraient expliquer pourquoi le Dr. Shapiro, avec beaucoup de perspicacité, a baptisé cette technique de mouvements oculaires désensibilisation et retraitement.

En effet, l'appui que notre étude apporte à l'augmentation de la cohérence interhémisphérique ne nie pas du tout les nombreux autres modèles offerts pour expliquer les mécanismes opératifs de l'EMDR. Il existe probablement de multiples mécanismes sous-jacents à l'efficacité de l'EMDR, car une intervention aussi puissante cliniquement et un cerveau aussi virtuellement infini dans son potentiel sont probablement trop complexes pour être expliquées par les propositions d'un seul modèle. Cette hyperbole mise à part, nous fournissons ici un timide soutien à l'élaboration d'un de ces premiers modèles cherchant à expliquer l'efficacité de l'EMDR, la CIh, en en proposant l'élargissement afin d'y inclure des régions corticales fonctionnelles spécifiquement en rapport avec le retraitement thérapeutique de souvenirs identifiés. En cohérence avec les résultats de recherche de Lyle et coll. (Edlyn \& Lyle, 2013 ; Lyle \& Jacobs, 2010 ; Lyle \& Martin, 2010 ; Lyle \& Osborn, 2011) qui suggèrent une traduction principalement intrahémisphérique de l'amélioration cognitive induite par les mouvements oculaires (ACIMO), nous proposons une approche autour de la cohérence corticale dans laquelle des voies corticales diffuses, spécifiques au type de stimulation bilatérale employée (visuelle, auditive, kinesthésique, etc.), établissent un niveau d'activation plus élevé ; ces voies sont alors plus facilement activées au cours du retraitement subséquent de l'événement cible (souvenirs positifs, négatifs ou traumatiques). Cette activation peut impliquer l'activation de réseaux neuronaux, soit entre les hémisphères (interhémisphériques), qui se manifesterait ensuite à travers l'augmentation de la CIh, soit à l'intérieur des hémisphères (intrahémisphériques), qui se refléterait dans l'augmentation de la cohérence dans 
des régions corticales plus localisées. Bien sûr, cette suggestion est basée sur une étude EEG plutôt limitée quant au nombre de participants, portant sur une population non clinique se rappelant des souvenirs positifs, et doit donc être extrêmement prudente à ce stade. Cependant, nous espérons que nos résultats et nos spéculations théoriques stimuleront des études de suivi afin de tester davantage nos hypothèses.

Dans notre étude portant sur des souvenirs émotionnels positifs, comme cela se produirait probablement lors de l'installation de ressources ou du Lieu Sûr dans les premières étapes de l'EMDR, des activités cognitives ne nécessitant peut-être pas de traitement additionnel et l'implication consécutive de réseaux neuronaux dissociés ou éloignés, il semble que des réseaux neuronaux droits et gauches plutôt circonscrits aient été activés. Il faudrait investiguer des souvenirs négatifs ou traumatiques, ayant encore à être complètement traités et intégrés, pour voir l'activation de réseaux plus nombreux et plus éloignés dans le souvenir ciblé à travers ces voies de stimulation bilatérale et, donc, des augmentations de cohérence intra- et interhémisphériques, à l'intérieur de régions corticales spécifiques, et entre elles. Cette hypothèse élargie reste encore à examiner plus globalement, mais nous espérons que les recherches en cours d'analyse dans nos laboratoires éclaireront davantage les mécanismes que nous avançons ici.

\section{Notes}

1. Habituellement, nous n'utilisons pas de procédures artéfactantes d'analyse automatique des composants indépendants (ICA)/d'analyse des composants principaux (PCA) dans notre laboratoire EEG parce que plus d'une dizaine d'années d'expérience nous ont montré que lorsque nous utilisons ce logiciel, nous devons tout de même procéder à un suivi avec des artéfacts visuels afin d'enlever les résidus d'artéfacts sonores. À la place, nous avons choisi d'adopter un protocole d'artéfact écrit détaillé, et le second auteur de cette étude (LS) conduit chaque année un atelier pratique avec des assistants de recherche, dans lequel ces critères sont émis et vérifiés avec de véritables données, afin de voir s'ils ont été suivis. De plus, tous les fichiers EEG sont " aveugles " et artéfactés deux fois afin de s'assurer que nos fichiers de données sont propres de tout bruit non EEG. Notre protocole est disponible, à la demande, auprès du second auteur (LS).

2. En tant que contrôle supplémentaire de la possible contribution des artéfacts musculaires des mouvements oculaires pour les effets EEG du pôle frontal, ces analyses ont été reconduites après avoir ôté les électrodes Fp1 et Fp2. Le même pattern d'effets significatifs et tendanciels a été obtenu dans cette nouvelle analyse.

\section{Bibliographie}

American Psychiatric Association. (2004). Practice guidelines for the treatment of patients with acute stress disorder and posttraumatic stress disorder. Arlington, VA : auteur.

Andrade, J., Kavanagh, D. \& Baddeley, A. (1997). Eye movements and visual imagery: A working memory approach to the treatment of post-traumatic stress disorder. British Journal of Clinical Psychology, 36, 209-223.

Bergmann, U. (2008). The neurobiology of EMDR: Exploring the thalamus and neural integration. Journal of EMDR Practice and Research, 2(4), 300-314.

Bisson, J. I., Ehlers, A., Mathews, R., Pilling, S., Richards, D. \& Turner, S. (2007). Psychological treatments for chronic post-traumatic stress disorder: Systematic review and meta-analysis. British Journal of Psychiatry, 190, 97-104.

Christman, S. D., Garvey, K. J., Propper, R. E. \& Phaneuf, K. A. (2003). Bilateral eye movements enhance the retrieval of episodic memories. Neuropsychology, 17, 221-229.

Christman, S. D. \& Propper, R. E. (2001). Superior episodic memory is associated with interhemispheric processing. Neuropsychology, 15, 607-616.

Christman, S. D., Propper, R. E. \& Brown, T. J. (2006). Increased interhemispheric interaction is associated with earlier offset of childhood amnesia. Neuropsychology, 20, 336-345.

Christman, S. D., Propper, R. E. \& Dion, A. (2004). Increased interhemispheric interaction is associated with decreased false memories in a verbal converging semantic associates paradigm. Brain and Cognition, 56, 313-319.

Cronin-Golomb, A., Gabrieli, J. D. E. \& Keane, M. M. (1996). Implicit and explicit memory retrieval within and across the disconnected cerebral hemispheres. Neuropsychology, 10, 254-262.

Cukor, J., Olden, M., Lee, F. \& Difede, J. (2010). Evidencebased treatments for PTSD, new directions, and special challenges. Annals of the New York Academy of Sciences, 1208, 82-89.

Davidson, P. R. \& Parker, K. C. H. (2001). Eye movement desensitization and reprocessing (EMDR): A metaanalysis. Journal of Consulting and Clinical Psychology, 69, 305-316.

Davidson, R. J. (1995). Cerebral asymmetry, emotion, and affective style. In R. J. Davidson \& K. Hugdahl (Eds.), Brain asymmetry (pp. 361-387). Cambridge, MA : MIT Press.

Davidson, R. J. (2002). Anxiety and affective style: Role of prefrontal cortex and amygdala. Biological Psychiatry, 51, 68-80.

Devilly, G. J. \& Spence, S. H. (1999). The relative efficacy and treatment distress of EMDR and a cognitivebehavior trauma treatment protocol in the amelioration of posttraumatic stress disorder. Journal of Anxiety Disorders, 13, 131-157.

Edlin, J. M. \& Lyle, K. B. (2013). The effect of repetitive saccade execution on the attention network test: Enhancing 
executive function with a flick of the eyes. Brain and Cognition, 81, 345-351.

Electro-Cap System[Appareil]. (1983). Eaton, Ohio:ElectroCap International.

Engelhard, I. M., van Uijen, S. L., \& van den Hout, M. A. (2010). The impact of taxing working memory on negative and positive memories. European Journal of Psychotraumatology, 1, 1-8.

EyeScan 2000S Light Bar [Appareil]. (1994). Wheat Ridge, Colorado : NeuroTek Corporation.

Francati, V., Vermetten, E. \& Bremner, J. D. (2007). Functional neuroimaging studies in posttraumatic stress disorder: Review of current methods and findings. Depression and Anxiety, 24, 202-218.

Gunter, R. W. \& Bodner, G. E. (2008). How eye movements affect unpleasant memories: Support for a workingmemory account. Behaviour Research and Therapy, 46, 913-931.

Harper, M. (2012). Taming the amygdala: An EEG analysis of exposure therapy for the traumatized. Traumatology, 18(2), 61-74.

Harper, M. L., Rasolkhani-Kaohorn, T. \& Drozd, J. F. (2009). On the neural basis of EMDR therapy: Insights from qEEG studies. Traumatology, 15, 81-95.

Hornsveld, H. K., Houtveen, J. H., Vroomen, M., Kapteijn, I., Aalbers, D. \& Van den Hout, M. A. (2011). Evaluating the effect of eye movements on positive memories such as those used in resource development and installation. Journal of EMDR Practice and Research, 5(4), 146-155.

Ironson, G., Freud, B., Strauss, J. L. \& Williams, J. (2002). Comparison of two treatments for traumatic stress: A community-based study of EMDR and prolonged exposure. Journal of Clinical Psychology, 58, 113-128.

Klimesch, W. (1999). EEG alpha and theta oscillations reflect cognitive and memory performance: A review and analysis. Brain Research Reviews, 29, 169-195.

Klimesch, W., Sauseng, P. \& Hanslmayr, S. (2007). EEG alpha oscillations: The inhibition-timing hypothesis. Brain Research Reviews, 53, 63-88.

Knott, V. J., LaBelle, A., Jones, B. \& Mahoney, C. (2002). EEG coherence following acute and chronic clozapine in treatment-resistant schizophrenics. Experimental and Clinical Psychopharmacology, 10(4), 435-444.

Knyazev, G. G. (2012). EEG delta oscillations as a correlate of basic homeostatic and motivational processes. Neuroscience and Biobehavioral Reviews, 36, 677-695.

Lamprecht, F., Kohnke, C., Lempa, W., Sack, M., Matzke, M. \& Munte, T. E. (2004). Event-related potentials and EMDR treatment of post-traumatic stress disorder. Neuroscience Research, 49, 267-272.

Lee, C. W. \& Drummond, P. D. (2008). Effects of eye movement versus therapist instructions on the processing of distressing memories. Journal of Anxiety Disorders, 22, 801-808.

Lee, C., Gavriel, H., Drummond, P., Richards, J. \& Greenwald, R. (2002). Treatment of PTSD: Stress inoculation training with prolonged exposure compared to EMDR. Journal of Clinical Psychology, 58, 1071-1089.
Lexicor NRS-24C [Appareil]. (1989). Boulder, Colorado : Lexicor Medical Technology.

LORETA: Low Resolution Electromagnetic Tomographic Analysis [Logiciel]. Zurich, Suisse : The KEY Institute for Brain-Mind Research.

Lyle, K. B. \& Jacobs, N. E. (2010). Is saccade-induced retrieval enhancement a potential means of improving eyewitness evidence? Memory, 18(6), 581-594.

Lyle, K. B., Logan, J. M. \& Roediger, H. L. (2008). Eye movements enhance memory for individuals who are strongly right-handed and harm it for individuals who are not. Psychonomic Bulletin and Review, 15, 515-520.

Lyle, K. B. \& Martin, J. M. (2010). Bilateral saccades increase intrahemispheric processing but not interhemispheric interaction: Implications for saccade-induced retrieval enhancement. Brain and Cognition, 73, 128-134.

Lyle, K. B. \& Orsborn, A. E. (2011). Inconsistent handedness and saccade execution benefit face memory without affecting interhemispheric interaction. Memory, 19(6), 613-624.

Maxfield, L., Melnyk, W. T. \& Hayman, C. A. G. (2008). A working memory explanation for the effects of eye movements in EMDR. Journal of EMDR Practice of Research, 2(4), 247-261.

MHyT [Appareil et logiciel]. (2000). Mesa, Arizona : Nova Tech EEG.

Neurosearch-24 V4.1E [Logiciel]. (1989). Boulder, Colorado : Lexicor Medical Technology.

Nova Tech EEG EureKa! [Appareil et logiciel]. (2000). Mesa, Arizona : Nova Tech EEG.

Nunez, P. L. \& Srinivasan, R. (2006). Electric fields in the brain: The neurophysis of EEG (2nde éd.). New York, NY : Oxford University Press.

Nunez, P. L., Srinivasan, R., Westdorp, A. F., Wijesinghe, R. S., Tucker, D. M., Silberstein, R. B. \& Cadusch, P. J. (1997). EEG coherency I: Statistics, reference electrode, volume conduction, Laplacians, cortical imaging, and interpretation at multiple sites. Electroencephalography and Clinical Neurophysiology, 103, 499-515.

Oldfield, R. (1971). The assessment and analysis of handedness: The Edinburgh inventory. Neuropsychologia, 9, 97-113.

Pagani, M., Hogberg, G., Fernandez, I. \& Siracusano, A. (2013). Correlates of EMDR therapy in functional and structural neuroimaging: A critical summary of recent findings. Journal of EMDR Practice and Research, 7(1), 29-38.

Parker, A., Buckley, S. \& Dagnall, N. (2009). Reduced misinformation effects following saccadic bilateral eye movements. Brain and Cognition, 69, 89-97.

Pascual-Marqui, R. D., Esslen, M., Kochi, K. \& Lehmann, D. (2002). Functional imaging with low resolution brain electromagnetic tomography (LORETA): A review. Methods and Findings in Experimental and Clinical Pharmacology, 24 (Suppl. C), 91-95.

Power, K., McGoldrick, T., Brown, K., Buchanan, R., Sharp, D. \& Swanson, V. (2002). A controlled comparison of eye movement desensitization and reprocessing versus exposure plus cognitive restructuring versus waiting list 
in the treatment of posttraumatic stress disorder. Clinical Psychology and Psychotherapy, 9, 299-318.

Propper, R. E. \& Christman, S. D. (2008). Interhemispheric interaction and saccadic horizontal eye movements: Implications for episodic memory, EMDR, and PTSD. Journal of EMDR Practice and Research, 2, 269-281.

Propper, R. E., Pierce, J., Geisler, M. W., Christman, S. D. \& Bellorado, N. (2007). Effect of bilateral eye move-ments on frontal interhemispheric gamma EEG coherence: Implications for EMDR therapy. The Journal of Nervous and Mental Disease, 195(9), 785-788.

Ramnani, N. \& Owen, A. M. (2004). Anterior prefrontal cortex: Insights into function from anatomy and neuroimaging. Nature Reviews: Neuroscience, 5, 184-194.

Rothbaum, B. O., Astin, M. C. \& Marsteller, F. (2005). Prolonged exposure versus eye movement desensitization and reprocessing (EMDR) for PTSD rape victims. Journal of Traumatic Stress, 18, 607-616.

Samara, Z., Elzinga, B. M., Slagter, H. A. \& Nieuwenhuis, S. (2011). Do horizontal saccadic eye movements increase interhemispheric coherence? Investigation of a hypothesized neural mechanism underlying EMDR. Frontiers in Psychiatry, 2(4), 1-9.

Servan-Schreiber, D. (2000). Eye movement desensitization and reprocessing: Is psychiatry missing the point? Psychiatric Times, 17, 36-40.

Shapiro, F. (1989). Efficacy of the eye movement desensitization procedure in the treatment of traumatic memories. Journal of Traumatic Stress Studies, 2, 199-223.

Shapiro, F. (2001). Eye movement desensitization and reprocessing: Basic principles, protocols, and procedures (2nde éd.). New York, NY : Guilford Press.

Shapiro, F. \& Maxfield, L. (2002). Eye movement desensitization and reprocessing (EMDR): Information processing in the treatment of trauma. Journal of Clinical Psychology, 58, 933-946.

Shobe, E. R., Ross, N. M. \& Fleck, J. I. (2009). Influence of handedness and bilateral eye movements on creativity. Brain and Cognition, 71, 204-214.

Solomon, R. M. \& Shapiro, F. (2008). EMDR and the adaptive information processing model: Potential mechanism for change. Journal of EMDR Practice and Research, 2(4), 315-325.

Stevens, L. C., Brady, B., Goon, A., Adams, D., Rebarchik, J., Gacula, L., . . . Verdugo, S. (2004). Electrophysiological alterations during hypnosis for ego-enhancement: A preliminary investigation. American Journal of Clinical Hypnosis, 46(4), 323-344.

Tabachnick, B. G. \& Fidell, L. S. (2013). Using multivariate statistics ( $6^{\mathrm{e}}$ éd.). Upper Saddle River, NJ: Pearson Education.

Taylor, S., Thordarson, D. S., Maxfield, L., Fedoroff, I. C., Lovell, K. \& Orgodniczuk, J. (2003). Comparative efficacy, speed, and adverse effects of three PTSD treatments: Exposure therapy, EMDR, and relaxation training. Journal of Consulting and Clinical Psychology, 71, 330-338.

Thatcher, R. W., Biver, C. J. \& North, D. (2004). EEG coherence and phase delays: Comparisons between single reference, average reference, and current source density. Manuscrit non publié, NeuroImaging Lab, VA Medical Center, Bay Pines, FL. Téléchargé depuis http: / / www.appliedneuroscience.com/ COMPARISONS-COMMONREF-AVE-LAPLACIAN .pdf 64

Trans Cranial Technologies. (2012). Cortical functions. Kowloon, Hong Kong, China: Trans Cranial Technologies, Ltd.

Tullett, A. M., Harmon-Jones, E. \& Inzlicht, M. (2012). Right frontal cortical asymmetry predicts empathic reactions: Support for a link between withdrawal motivation and empathy. Psychophysiology, 49, 1145-1153.

Tulving, E. (1985). How many memory systems are there? American Psychologist, 40(4), 385-398.

Van den Hout, M. A., Eidhof, M. B., Verboom, J., Littel, M. \& Engelhard, I. M. (2013). Blurring of emotional and non-emotional memories by taxing working memory during recall. Cognition \& Emotion, 1-10.

Van den Hout, M. A. \& Engelhard, I. M. (2012). How does EMDR work? Journal of Experimental Psychopathology, 3(5), 724-738.

Van den Hout, M. A., Muris, P., Salemink, E. \& Kindt, M. (2001). Autobiographical memories become less vivid and emotional after eye movements. British Journal of Clinical Psychology, 40, 121-130.

Weiss, S. \& Mueller, H. M. (2003). The contribution of EEG coherence to the investigation of language. Brain and Language, 85, 325-343.

Remerciements. Cette étude a été menée par le premier auteur en tant que projet de fin d'études, sous la supervision du second. Des parties en ont été présentées par le premier auteur à la $5^{\mathrm{e}}$ convention annuelle Psi Chi de recherche de fin de diplôme, à Flagstaff (Arizona), le 10 avril 2010 ; à la $40^{\mathrm{e}}$ rencontre annuelle de la Société pour les neurosciences, à San Diego (Californie), le 14 novembre 2010 ; et enfin à la $119^{\mathrm{e}}$ convention annuelle de la Société américaine de psychologie (APA), à Washington DC, le 3 août 2011, et par le cinquième auteur à la $91^{\mathrm{e}}$ Convention annuelle de la Société de psychologie à Los Angeles (Californie), le 30 avril 2011.

Merci d'adresser toute correspondance concernant cet article à Dr Larry Stevens, Northern Arizona University, Department of Psychological Sciences, Box 15106, Flagstaff, AZ 86011, États-Unis. Courriel : larry.stevens@nau.edu 